\title{
Specht modules with abelian vertices
}

\author{
Kay Jin Lim
}

Received: 13 February 2011 / Accepted: 1 June 2011 / Published online: 28 June 2011

(C) Springer Science+Business Media, LLC 2011

\begin{abstract}
In this article, we consider indecomposable Specht modules with abelian vertices. We show that the corresponding partitions are necessarily $p^{2}$-cores where $p$ is the characteristic of the underlying field. Furthermore, in the case of $p \geq 3$, or $p=2$ and $\mu$ is 2-regular, we show that the complexity of the Specht module $S^{\mu}$ is precisely the $p$-weight of the partition $\mu$. In the latter case, we classify Specht modules with abelian vertices. For some applications of the above results, we extend a result of M. Wildon and compute the vertices of the Specht module $S^{\left(p^{p}\right)}$ for $p \geq 3$.
\end{abstract}

Keywords Specht module $\cdot$ Vertex $\cdot$ Complexity

\section{Introduction}

The representation theory of symmetric groups has been one of the major research areas since the beginning of the last century. The theory has been well-developed, yet it seems that very little is known for the modular case. One way of understanding the structure of representations of finite groups is through the notion of relative projectivity, on which J.A. Green [13] defined the vertices of modules of finite groups about 50 years ago. The vertices of modules are, in some way, related to the complexity of the modules defined by J. Alperin and L. Evens [2], and the rank variety defined by J. Carlson [6].

Classically, Young modules, Specht modules and simple modules are the major objects extensively studied in the representation theory of symmetric groups. The computation of the vertices of Young modules has been done by J. Grabmeier [12].

K.J. Lim (ه)

Department of Mathematics, National University of Singapore, Block S17, 10 Lower Kent Ridge

Road, Singapore 119076, Singapore

e-mail: matlkj@nus.edu.sg 
The vertices of signed Young modules have been computed by S. Donkin [9]. However, the vertices of simple modules and Specht modules remain mostly unknown. The vertices of Specht modules of hook shape in $p=2$ case were first considered by G.M. Murphy and M.H. Peel in [24], with a mistake which has been corrected by M. Wildon [27]. M. Wildon has made some progress on the computation of the vertices of Specht modules, namely he has computed the vertices of simple Specht modules of hook shape [27] and showed that, in general, a vertex of a Specht module contains some large $p$-subgroup [28]. Recently, there were some computation of the vertices of simple modules made by S. Danz, B. Külshammer, J. Müller and R. Zimmermann [8, 23].

It is well known that, in general, if the defect groups of an indecomposable module are abelian then its vertices are necessarily abelian, too. In particular, for the representations of symmetric groups in the modular case, representations with abelian defect correspond to partitions of $p$-weights strictly less than the characteristic $p$ of the underlying field. But there are examples of Specht modules of hook shape whose vertices are abelian, yet their defect groups are not abelian. For example, for $p=3$ the Specht module $S^{\left(7,1^{3}\right)}$ has vertices the Sylow 3-subgroups of $\mathfrak{S}_{6} \times \mathfrak{S}_{3}$ (see [27, Theorem 2]) but with defect groups the Sylow 3-subgroups of $\mathfrak{S}_{9}$.

In this article, we shall mainly be concerned with Specht modules with abelian vertices. Motivated by the question of classifying these Specht modules, we give some necessary conditions for such Specht modules. Indeed, for $p \geq 3$, we show that no other abelian subgroups can be the vertices of Specht modules besides the elementary abelian ones. For $p=2$, we could not give a definite answer as in the odd characteristic case unless the corresponding partitions are 2-regular. Under the hypothesis that the vertices of the Specht modules are abelian, a class of partitions arises naturally, namely the $p^{2}$-core partitions. In fact, for $p=2$, we show that a 2 regular partition $\mu$ is a 4-core if and only if the Specht module $S^{\mu}$ has elementary abelian vertices. For all the Specht modules mentioned above, we conclude that their complexities are precisely the $p$-weights of their labeled partitions.

We organize the article in the following way. In Sect. 2, we lay down some basic knowledge about the representations of symmetric groups, the complexities and the rank varieties for modules. We state our main results in Sect. 3 and prove them in Sect. 4. In Sect. 5, we draw some consequences of the main results, in which we generalize a result of M. Wildon and show that the vertices of $S^{\left(p^{p}\right)}$ are the Sylow $p$-subgroups of $\mathfrak{S}_{p^{2}}$ when $p \geq 3$. This is an example where the partition $\left(p^{p}\right)$ is a $p^{2}$-core but the Specht module $S^{\left(p^{p}\right)}$ does not have abelian vertices. In the last section Sect. 6, we post some questions which arise naturally from our results.

\section{Preliminaries}

We introduce the notation and background which we require. General references for this section are $[4,16,18]$.

\subsection{The representations}

Let $G$ be a finite group and $F$ be a field of characteristic $p$. In this article, all $F G$ modules are finite dimensional vector spaces over $F$. 
Let $M$ be an $F G$-module and $H$ be a subgroup of $G$. We regard the restriction $\operatorname{Res}_{H}^{G} M$ as the $F H$-module in the obvious way. If $S$ is an $F H$-module, we write $\operatorname{Ind}_{H}^{G} S$ for the induced module. Let $N$ be another $F G$-module. We write $N \mid M$ if $N$ is isomorphic to a direct summand of $M$ as an $F G$-module.

Suppose that $p>0$. We say that the module $M$ is relatively $H$-projective if $M \mid \operatorname{Ind}_{H}^{G} \operatorname{Res}_{H}^{G} M$. A vertex $Q$ of an indecomposable $F G$-module $M$ is a minimal subgroup of $G$ subject to the condition that $M$ is relatively $Q$-projective. Given a vertex $Q$ of $M$, a source of $M$ is an indecomposable $F Q$-module $S$ such that $M \mid \operatorname{Ind}_{Q}^{G} S$.

Let $F G=I_{1} \oplus \cdots \oplus I_{m}$ be a decomposition of the $F(G \times G)$-module $F G$, with the action given by $(g, h) x=g x h^{-1}$, into indecomposables. Suppose that $1=\sum_{i=1}^{m} e_{i}$ with $e_{i} \in I_{i}$ for each $1 \leq i \leq m$. Indeed, $I_{i}=e_{i} F G$. The elements $e_{i}$ are mutually orthogonal primitive central idempotents of the algebra $F G$ and they are called the blocks of $F G$. For an indecomposable $F G$-module $M$, there is a unique block $e_{j}$ such that $e_{j} M \neq 0$ and $e_{i} M=0$ for all $i \neq j$. In this case, $e_{j} M=M$ and we say that the module $M$ lies in the block $e_{j}$. Note that $F G \cong \operatorname{Ind}_{\Delta(G)}^{G \times G} F$ where $\Delta(G)$ is the diagonal embedding of $G$ into $G \times G$. Thus, for each $1 \leq i \leq m$, a vertex of $I_{i}$ is of the form $\Delta\left(D_{i}\right)$ for some subgroup $D_{i}$ of $G$ and the subgroup $D_{i}$ is called a defect group of the block $e_{i}$.

Theorem 2.1 ([13]) Let $F$ be a field of characteristic $p>0, G$ be a finite group and $M$ be an indecomposable $F G$-module. Then

(i) Any vertex of $M$ is a p-subgroup of $G$,

(ii) The vertices of $M$ are conjugate to each other in $G$, and

(iii) If $M$ lies in the block $e_{j}$ of $F G$ then $M$ is relatively $D_{j}$-projective where $D_{j}$ is a defect group of $e_{j}$. In particular, a vertex of $M$ is necessarily a subgroup of $D_{j}$ up to conjugation.

Suppose that $L$ is a field extension of $F$. We write $L \otimes_{F} M$ for the $L G$-module upon field extension. In the latter part of this article, we are required to deal with vertices of modules over field extension. We include a little lemma here.

Lemma 2.2 ([11, Sect. III Lemma 4.14]) Let L be a field extension of $F$ and $H$ be a p-subgroup of a finite group $G$. Then an $F G$-module $M$ is relatively $H$-projective if and only if $L \otimes_{F} M$ is relatively $H$-projective.

In particular, if $M$ is indecomposable and $L \otimes_{F} M$ remains indecomposable as an $L G$-module then a $p$-subgroup $Q$ of $G$ is a vertex of $M$ if and only if $Q$ is a vertex of $L \otimes_{F} M$.

\subsection{Rank varieties}

Let $p$ be a prime. The $p$-rank of a finite group $G$ is the largest integer $n$ subject to the condition that $G$ contains an elementary abelian $p$-subgroup of order $p^{n}$. The $p$-rank of the abelian $p$-group $\mathbb{Z}_{p^{n_{1}}} \times \cdots \times \mathbb{Z}_{p^{n_{m}}}$ with $n_{1}, \ldots, n_{m}>0$ is $m$.

Let $E$ be an elementary abelian $p$-group of $p$-rank $n$ with generators $g_{1}, \ldots, g_{n}$. For each non-zero point $\alpha=\left(\alpha_{1}, \ldots, \alpha_{n}\right)$ of the space $F^{n}$, we write $u_{\alpha}=1+$ 
$\sum_{i=1}^{n} \alpha_{i}\left(g_{i}-1\right) \in F E$. Note that $\left\langle u_{\alpha}\right\rangle$ is a cyclic $p$-group. Suppose that $F$ is algebraically closed. The rank variety of a $F E$-module $M$ is the set

$$
V_{E}^{\#}(M)=\{\mathbf{0}\} \cup\left\{\mathbf{0} \neq \alpha \in F^{n} \mid M \text { is not projective as } F\left\langle u_{\alpha}\right\rangle \text {-module }\right\} .
$$

The rank variety $V_{E}^{\#}(M)$ is a homogeneous and closed subvariety of the affine space $\mathbb{A}^{n}(F)$ [6, Theorem 4.3]. We shall write $\operatorname{dim} V_{E}^{\#}(M)$ for the dimension of the algebraic variety $V_{E}^{\#}(M)$. The rank variety depends on the choice and order of the generators of $E$, but its dimension does not. In the case where $p \nmid \operatorname{dim}_{F} M$, for each $\mathbf{0} \neq \alpha \in$ $F^{n}, M$ necessarily has a summand of dimension coprime to $p$ as $F\left\langle u_{\alpha}\right\rangle$-modules. In this case, we have $V_{E}^{\#}(M)=V_{E}^{\#}(F)$ and thus $\operatorname{dim} V_{E}^{\#}(M)=n$. Let $N$ be another $F E$-module. It is clear from the definition that $V_{E}^{\#}(M \oplus N)=V_{E}^{\#}(M) \cup V_{E}^{\#}(N)$.

Let $G$ be a finite group and $M$ be a $F G$-module. Let

$$
\mathbf{P}: \cdots \rightarrow P_{2} \rightarrow P_{1} \rightarrow P_{0} \rightarrow M
$$

be a minimal projective resolution of $M$. Following [2], the complexity $c$ of the module $M$ is the smallest non-negative integer such that

$$
\lim _{n \rightarrow \infty} \frac{\operatorname{dim}_{F} P_{n}}{n^{c}}=0
$$

in other words, the polynomial rate growth of the dimensions of the terms of the sequence $\mathbf{P}$. We write $c_{G}(M)$ for the number $c$ to indicate the dependence of $c$ on $M$ as an $F G$-module.

\section{Theorem 2.3}

(i) ([2, Sect. 1 Theorem], [3, Theorem 1.1] and [6, Theorem 7.6]) Let $M$ be an $F G$ module, $\mathcal{E}$ be a set of representatives of elementary abelian p-subgroups of $G$ up to conjugation and $\mathcal{E}^{\max }$ be a set of representatives of maximal elementary abelian p-subgroups of $G$ up to conjugation. Then

$$
c_{G}(M)=\max _{E \in \mathcal{E}}\left\{\operatorname{dim} V_{E}^{\#}(M)\right\}=\max _{E \in \mathcal{E}^{\max }}\left\{\operatorname{dim} V_{E}^{\#}(M)\right\} .
$$

(ii) [2, Corollary 4, 5] The complexity of an indecomposable FG-module $M$ is bounded above by the p-rank of a defect group of the block in which M lies.

Following Theorem 2.3 (i), we have the conclusion that $c_{G}(M)$ is the $p$-rank of $G$ provided $p \nmid \operatorname{dim}_{F} M$. We have a more refined statement of Theorem 2.3 (ii).

Proposition 2.4 [2, Lemmas 5.2, 5.3] Let $M$ be an FG-module where $F$ is a field of characteristic $p>0$.

(i) Suppose that $M$ is relatively $H$-projective for some subgroup $H$ of $G$. Then $c_{G}(M)=c_{H}(M)$.

(ii) Suppose that $M$ is indecomposable. Let $Q$ be a vertex of $M$ with a source $S$. Then $c_{G}(M)=c_{Q}(S)$. 
Readers who are familiar with the variety theory for modules would have realized that the author has avoided taking cohomological variety into discussion, thanks to the Quillen stratification theorem and [3, Theorem 1.1].

\subsection{The representations of symmetric groups}

We now briefly go through the representations of symmetric groups. Let $n$ be a nonnegative integer. A partition $\mu$ of $n$ is a sequence of positive integers $\left(\mu_{1}, \mu_{2}, \ldots, \mu_{s}\right)$ such that $\mu_{1} \geq \mu_{2} \geq \cdots \geq \mu_{s}$ and $\sum_{i=1}^{s} \mu_{i}=n$. In this case, we write $|\mu|=n$. Note that we allow the empty partition $\varnothing$ to be the unique partition of 0 . The partition $\mu$ is called $p$-singular if there is some $i \geq 0$ such that $\mu_{i+1}=\mu_{i+2}=\cdots=\mu_{i+p}>0$; otherwise, it is called $p$-regular. Let $\Lambda(n)$ be the set consisting of all partitions of $n$. There is a one-to-one correspondence between $\Lambda(n)$ and the set of Young diagrams with $n$ nodes in an obvious way. The Young diagram of $\mu$ is written as $[\mu]$. Fix a positive integer $m$, not necessarily a prime. Each partition $\mu$ is associated to a partition $\tilde{\mu}$ and an non-negative integer $w$ such that $|\mu|=|\tilde{\mu}|+m w$. The partition $\widetilde{\mu}$ and the integer $w$ is called the $m$-core and the $m$-weight of $\mu$, respectively [16, Sect. 2.7]. The $m$-core of $\mu$ is obtained by successively removing $w$ removable rim $m$-hooks. In the case where $w=0$, or equivalently $\mu=\tilde{\mu}$, we say that the partition $\mu$ is an $m$-core.

We write $\mathfrak{S}_{n}$ for the symmetric group acting on $n$ letters. To each partition $\mu$ of $n$, we have the $F \mathfrak{S}_{n}$-module $S_{F}^{\mu}$, the Specht module labeled by the partition $\mu$. We usually write $S^{\mu}$ for $S_{F}^{\mu}$ if the underlying field is understood. The dimension of the Specht module $S_{F}^{\mu}$ is given by the hook formula

$$
\operatorname{dim}_{F} S_{F}^{\mu}=\frac{n !}{\prod_{(i, j) \in[\mu]} h_{i, j}}
$$

where $h_{i, j}$ denotes the hook length of the node $(i, j)$ of $[\mu]$ and $n=|\mu|$. We note that the dimension of $S_{F}^{\mu}$ is independent of the field $F$.

Theorem 2.5 Let $p$ be the characteristic of a field $F$ and $n$ be a positive integer.

(i) $[16,2.7 .40]$ Let $m$ be a positive integer. The number of hook lengths of a Young diagram $[\mu]$ which are divisible by $m$ is precisely the $m$-weight of $\mu$.

(ii) [18, Theorem 4.12] For $p=0$, the set $\left\{S^{\mu} \mid \mu \in \Lambda(n)\right\}$ is a complete set of nonisomorphic simple $F \mathfrak{S}_{n}$-modules.

(iii) [18, Corollary 13.18] Let $\mu$ be a partition. For $p \geq 3$, or $p=2$ and $\mu$ is 2regular, the Specht module $S^{\mu}$ is indecomposable.

(iv) [16, Sect. 6.1 and Theorem 6.2.45] The blocks of $F \mathfrak{S}_{n}$ are parametrized by the p-cores of the partitions of $n$ such that the block $e_{\tilde{\mu}}$ labeled by the p-core $\tilde{\mu}$ contains the Specht module $S^{\mu}$. In particular, if $\mu, \lambda$ are partitions of $n$ then the Specht modules $S^{\mu}, S^{\lambda}$ lie in the same block of $F \mathfrak{S}_{n}$ if and only if $\tilde{\mu}=\tilde{\lambda}$. A defect group of the block $e_{\tilde{\mu}}$ is conjugate to a Sylow p-subgroup of $\mathfrak{S}_{w p}$ where $w$ is the p-weight of $\mu$.

We shall take a step further to discuss the vertices of a special class of modules of the symmetric groups, the Young modules [19]. Here and hereafter, whenever we 
have subgroups $H \subseteq \mathfrak{S}_{a}, K \subseteq \mathfrak{S}_{b}$ where $a+b \leq n$ for some positive integers $a, b, n$, we write $H \times K \hookrightarrow \mathfrak{S}_{a} \times \mathfrak{S}_{b} \hookrightarrow \mathfrak{S}_{n}$ for the obvious inclusions.

Let $\mu=\left(\mu_{1}, \ldots, \mu_{r}\right)$ be a partition of $n$. Let $\mathfrak{S}_{\mu}$ be the Young subgroup of $\mathfrak{S}_{n}$, i.e.,

$$
\mathfrak{S}_{\mu}=\mathfrak{S}_{\mu_{1}} \times \mathfrak{S}_{\mu_{2}} \times \cdots \times \mathfrak{S}_{\mu_{r}}
$$

Let $M^{\mu} \cong \operatorname{Ind}_{\mathfrak{S}_{\mu}}^{\mathfrak{S}_{n}} F$ be the associated permutation module. Suppose that $M^{\mu}=M_{1} \oplus$ $\cdots \oplus M_{k}$ is a decomposition of $M^{\mu}$ into indecomposable summands. Then there is a unique direct summand $M_{j}$ of $M^{\mu}$ such that $S^{\mu} \subseteq M_{j}$. We write $Y^{\mu}$ for $M_{j}$ and call it a Young module. Clearly, the Young modules are well-defined up to isomorphism. Every indecomposable direct summand of $M^{\mu}$ is isomorphic to some Young module and $Y^{\mu} \cong Y^{\lambda}$ if and only if $\mu=\lambda$. The Young modules have trivial sources. It is well known that if $\mu$ is a $p$-regular partition and $S^{\mu}$ is a simple Specht module then $S^{\mu} \cong Y^{\mu} ;$ see, for example, [7, Sect. 1].

Every partition $\mu$ can be written as its $p$-adic expansion as follows. Let $\mu_{(0)}$ be the partition obtained from $\mu$ by successively stripping off all horizontal $p$-hooks. Then we have the coordinate-wise summation $\mu=p \mu(1)+\mu_{(0)}$ for some partition $\mu(1)$. Inductively, we can write $\mu(k-1)=p \mu(k)+\mu_{(k-1)}$ for $k \geq 2$ and hence $\mu=$ $p^{k} \mu(k)+\cdots+p \mu_{(1)}+\mu_{(0)}$. The process ends if for some $k, \mu(k)$ has no removable horizontal $p$-hooks. Let $\mu_{(k)}=\mu(k)$. Then $\mu=p^{k} \mu_{(k)}+\cdots+p \mu_{(1)}+\mu_{(0)}$ is the $p$-adic expansion of $\mu$. Let $\rho(\mu)$ be the partition

$$
(\underbrace{p^{k}, \ldots, p^{k}}_{\left|\mu_{(k)}\right| \text { factors }}, \ldots, \underbrace{p, \ldots, p}_{\left|\mu_{(1)}\right| \text { factors }}, \underbrace{p^{0}, \ldots, p^{0}}_{\left|\mu_{(0)}\right| \text { factors }})
$$

of $n$ and $\mathfrak{S}_{\rho(\mu)}$ be the corresponding Young subgroup of $\mathfrak{S}_{n}$.

Theorem 2.6 ([12], [10, Sect. 4.1]) Any vertex of the Young module $Y^{\mu}$ is conjugate to a Sylow p-subgroup of $\mathfrak{S}_{\rho(\mu)}$.

The main purpose of this article is to study the vertices and complexity of Specht modules. Thus, by Theorem 2.3 (i), it is crucial to understand the maximal elementary abelian $p$-subgroups of a symmetric group up to conjugation. Let $m$ be a positive integer. Let $\left(\mathbb{Z}_{p}\right)^{m}$ act on itself by the left regular action. This induces an injective group homomorphism $\left(\mathbb{Z}_{p}\right)^{m} \hookrightarrow \mathfrak{S}_{p^{m}}$. Since the action is faithful we write $V_{m}(p)$ for the image of this homomorphism.

Theorem 2.7 ([1, Sect. VI Theorem 1.3]) Let $n$ be a positive integer. There is a one-to-one correspondence between the ways of writing $n=i_{0}+i_{1} p+\cdots+i_{r} p^{r}$ for some non-negative integer $r$ such that all $i_{0}, \ldots, i_{r}$ are non-negative integers and $0 \leq i_{0} \leq p-1$, and the set of representatives of all maximal elementary abelian p-subgroups of $\mathfrak{S}_{n}$ up to conjugation, given by 


$$
\begin{aligned}
& \underbrace{V_{1}(p) \times \cdots \times V_{1}(p)}_{i_{1} \text { times }} \times \cdots \times \underbrace{V_{r}(p) \times \cdots \times V_{r}(p)}_{i_{r} \text { times }} \\
& \hookrightarrow \underbrace{\mathfrak{S}_{p} \times \cdots \times \mathfrak{S}_{p}}_{i_{1} \text { times }} \times \cdots \times \underbrace{\mathfrak{S}_{p^{r}} \times \cdots \times \mathfrak{S}_{p^{r}}}_{i_{r} \text { times }} \hookrightarrow \mathfrak{S}_{n} .
\end{aligned}
$$

Note that the $p$-rank of the maximal elementary abelian $p$-subgroup of $\mathfrak{S}_{n}$ given in Theorem 2.7 is $i_{1}+2 i_{2}+\cdots+r i_{r}$. We shall draw an easy conclusion, following Theorem 2.5 (iv) and Theorem 2.3 (ii), that the complexity of a Specht module $S^{\mu}$ is bounded above by the $p$-weight of $\mu$. The statement remains valid if we replace the Specht module by the simple module $D^{\mu}$, whenever $\mu$ is $p$-regular, of $F \mathfrak{S}_{n}$ as there is an analogous statement to Theorem 2.5 (iv) for simple modules, though this is not of the main concern of this article.

\section{Main results}

We present our main results in this section.

Theorem 3.1 Let $F$ be a field of characteristic $p>0$ and $S^{\mu}$ be an indecomposable Specht module. Suppose that $S^{\mu}$ has an abelian vertex of $p$-rank $m$. Then $\mu$ is a $p^{2}$-core and the complexity of $S^{\mu}$ is $m$.

Theorem 3.2 Let $F$ be a field of characteristic $p>0$ and $\mu$ be a partition. Suppose that $p \geq 3$, or $p=2$ and $\mu$ is 2-regular. Suppose that the Specht module $S^{\mu}$ has an abelian vertex $Q$ of p-rank $m$. Let c be the complexity of the Specht module $S^{\mu}$ and $w$ be the p-weight of the partition $\mu$. Then $c=m=w$ and $Q$ is conjugate to the elementary abelian p-subgroup

$$
\underbrace{V_{1}(p) \times \cdots \times V_{1}(p)}_{\text {factors }} \hookrightarrow \mathfrak{S}_{p} \times \cdots \times \mathfrak{S}_{p} \hookrightarrow \mathfrak{S}_{n} .
$$

Theorem 3.3 Let $p=2, \mu$ be a 2-regular partition and $Q$ be a vertex of the Specht module $S^{\mu}$. Let $w$ be a non-negative integer. Then the following statements are equivalent.

(i) The vertex $Q$ is abelian of 2-rank $w$.

(ii) The vertex $Q$ is elementary abelian of 2-rank $w$.

(iii) The partition $\mu$ is a 4-core of 2-weight $w$.

In any of these cases, the Specht module $S^{\mu}$ has trivial source, complexity $w$ and is a simple Young module.

Remark 3.4 The hypothesis of Theorem 3.3 cannot be loosened in the following sense. For $p=2$, it is known that the Specht modules $S^{\left(n-r, 1^{r}\right)}$ are indecomposable when $2 \mid n$ [25, Theorem 4.1]. In this case, the vertices of $S^{\left(n-r, 1^{r}\right)}$ are the Sylow 2 -subgroups of $\mathfrak{S}_{n}\left[24\right.$, Theorem 4.5]. Thus, for instance, the Specht module $S^{(4,1,1)}$ has a non-abelian vertex, yet the partition $(4,1,1)$ is a 4-core. So, in Theorem 3.3, we cannot replace the 2 -regularity condition merely by the indecomposability condition. 


\section{Proof of the main results}

In this section, we shall prove our main theorems. We first prove Theorem 3.1. We then divide the latter part of this section into two subsections. The first part concerns the proof of Theorem 3.2 when $p \geq 3$. The second part concerns the proofs of Theorem 3.2 when $p=2$ and Theorem 3.3.

We state two known results upon which our proofs rely heavily.

Theorem 4.1 ([26], [20, Theorem 1]) Let $n_{1}, \ldots, n_{s}$ be positive integers. If there is an injective group homomorphism $\mathbb{Z}_{p^{n_{1}}} \times \cdots \times \mathbb{Z}_{p^{n_{s}}} \hookrightarrow \mathfrak{S}_{m}$ then $p^{n_{1}}+\cdots+p^{n_{s}} \leq$ $m$.

For any positive integer $n$ and prime number $p$, we write $n_{p}$ for the prime power $p^{a}$ such that $p^{a} \mid n$ and $\operatorname{gcd}\left(p, n / p^{a}\right)=1$.

Theorem 4.2 ([5, Theorem 1.1]) Let $M$ be an FG-module for some finite group $G$. Suppose that $M$ is relatively $H$-projective for some subgroup $H$ of $G$. Let $Q \cong$ $\mathbb{Z}_{p^{n_{1}}} \times \cdots \times \mathbb{Z}_{p^{n_{m}}}$ be an abelian subgroup of $H$ of $p$-rank $m$ with $n_{1} \geq \cdots \geq n_{m} \geq 1$. Let $c=c_{Q}(M)$ be the complexity of the $F Q$-module $\operatorname{Res}_{Q}^{G} M$. Then

$$
\frac{|G: H|_{p}|Q|_{p}}{p^{n_{1}} \cdots p^{n_{c}}} \mid\left(\operatorname{dim}_{F} M\right)_{p} .
$$

We can now prove Theorem 3.1.

Proof of Theorem 3.1 We assume the notation as in Theorem 3.1. Let $n=|\mu|$ and $w$ be the $p$-weight of $\mu$. Let $D$ be a defect group of the block $e_{\tilde{\mu}}$ and $Q$ be a vertex of $S^{\mu}$. Without loss of generality, we may assume that

$$
Q \subseteq D \subseteq \mathfrak{S}_{p w}
$$

We apply Theorem 4.2 and Proposition 2.4 by taking $M=S^{\mu}, H=Q \cong \mathbb{Z}_{p^{n_{1}}} \times$ $\cdots \times \mathbb{Z}_{p^{n_{m}}}$ with $n_{1} \geq \cdots \geq n_{m} \geq 1$. Let $c=c_{Q}\left(S^{\mu}\right)=c_{\mathfrak{S}_{n}}\left(S^{\mu}\right)$. We have

$$
p^{a-n_{1}-\cdots-n_{m}} \cdot p^{n_{c+1}} \cdots p^{n_{m}}=p^{a-n_{1}-\cdots-n_{c}} \mid\left(\operatorname{dim}_{F} S^{\mu}\right)_{p}
$$

where $p^{a}=(n !)_{p}$. By Theorem 2.5 (i) and the hook formula, we let $s$ be the nonnegative integer such that

$$
p^{s}=\frac{p^{a}}{p^{w} \cdot\left(\operatorname{dim}_{F} S^{\mu}\right)_{p}}=\frac{\prod_{(i, j) \in[\mu]}\left(h_{i, j}\right)_{p}}{p^{w}} .
$$

Note that $s=0$ if and only if $\mu$ is a $p^{2}$-core; namely every hook length of $[\mu]$ is not divisible by $p^{2}$. With this notation, we deduce that

$$
s+w \leq n_{1}+\cdots+n_{c} .
$$


On the other hand, we have the injection $Q \hookrightarrow \mathfrak{S}_{p w}$. As such, by Theorem 4.1, we get $p^{n_{1}}+\cdots+p^{n_{m}} \leq p w$; namely $p^{n_{1}-1}+\cdots+p^{n_{m}-1} \leq w$. Combining this inequality with (4.1), we have

$$
\left(p^{n_{1}-1}-n_{1}\right)+\cdots+\left(p^{n_{c}-1}-n_{c}\right)+p^{n_{c+1}-1}+\cdots+p^{n_{m}-1}+s \leq 0 .
$$

Since $p^{k-1}-k \geq 0$ for every positive integer $k$ we conclude that $m=c, s=0$, $p^{n_{i}-1}=n_{i}$ for all $1 \leq i \leq c$ and $w=n_{1}+\cdots+n_{c}$. We have proved Theorem 3.1.

\subsection{The case where $p \geq 3$}

Assuming all the computations what we have just done in the proof of Theorem 3.1, we continue to prove Theorem 3.2 for the case of $p \geq 3$.

Proof of Theorem 3.2 when $p \geq 3$ If $p$ is odd then, from $p^{n_{i}-1}=n_{i}$, necessarily $n_{i}=1$ for all $1 \leq i \leq c$; namely $m=c=w$ and $Q$ is an elementary abelian $p$-group of $p$-rank $w$. Since $Q$ is also a subgroup of $\mathfrak{S}_{p w}$, by Theorem 2.7, we let

$$
E=\left(V_{1}(p)\right)^{i_{1}} \times \cdots \times\left(V_{r}(p)\right)^{i_{r}}
$$

be an maximal elementary $p$-subgroup of $\mathfrak{S}_{p w}$ containing $Q$. Comparing their $p$ ranks, we have $w \leq i_{1}+2 i_{2}+\cdots+r i_{r}$. On the other hand, we also have $i_{1} p+$ $i_{2} p^{2}+\cdots+i_{r} p^{r}=p w$. Thus we deduce that

$$
i_{2}(p-2)+\cdots+i_{r}\left(p^{r-1}-r\right) \leq 0
$$

and hence $i_{2}=\cdots=i_{r}=0, i_{1}=w$. Namely, $Q$ is necessarily conjugate to $\left(V_{1}(p)\right)^{w}$. This completes the proof of Theorem 3.2 when $p$ is odd.

Remark 4.3 The author would like to point out that the proofs given above, for Theorem 3.1 and Theorem 3.2 when $p \geq 3$, are very similar to the proof of [27, Theorem 1] in which Wildon dealt with the case where $m=1$.

\subsection{The case where $p=2$}

Note that the proof of Theorem 3.1 assumes nothing about the 2-regularity of the partition $\mu$, in the case of $p=2$. It works for all indecomposable Specht modules with abelian vertices. In view of Theorem 3.2, we still have a result for $p=2$ case without assuming the 2-regularity condition.

Proposition 4.4 Let $p=2$ and $S^{\mu}$ be indecomposable. Suppose that a vertex $Q$ of $S^{\mu}$ is abelian of $p$-rank $m$. Let $c$ be the complexity of $S^{\mu}$ and $w$ be the 2-weight of $\mu$. Then $m=c, c \leq w \leq 2 c$ and the direct factors of $Q$ are either $\mathbb{Z}_{2}$ or $\mathbb{Z}_{4}$.

Proof By (4.2) with $p=2$, we have $m=c, 2^{n_{i}-1}=n_{i}$ for all $1 \leq i \leq c$ and $w=$ $n_{1}+\cdots+n_{c}$. Thus each of the $n_{i}$ is either 1 or 2 . 
In general, we do not know when a Specht module over an even characteristic field is decomposable. Since vertices are defined only for indecomposable modules, in view of Theorem 2.5 (iii), the 2-regularity assumption is technically required.

Note that the proof which we have just given in Sect. 4.1 for Theorem 3.2 fails when $p=2$ because of the possibility that $Q$ may have $\mathbb{Z}_{4}$ as a direct factor. Thus we need to take a closer look at these partitions $\mu$ which are 2-regular and the vertices of $S^{\mu}$ are abelian.

Lemma 4.5 Let $\mu=\left(\mu_{1}, \ldots, \mu_{r}\right)$ be a partition. Then $\mu$ is both a 2-regular and 4-core partition if and only if exactly one of the following holds.

(i) $\mu_{r}=1$ and there exists a number $0 \leq s \leq r-1$ such that $\mu_{i}-\mu_{i+1}=3$ for all $1 \leq i \leq s$ and $\mu_{i}-\mu_{i+1}=1$ for all $s+1 \leq i \leq r-1$.

(ii) $\mu_{r} \in\{2,3\}$ and $\mu_{i}-\mu_{i+1}=3$ for all $1 \leq i \leq r-1$.

Proof Suppose that $\mu$ is 2-regular and is a 4-core. It is easy to see that $1 \leq \mu_{i}-$ $\mu_{i+1} \leq 3$ for all $1 \leq i \leq r-1$ and $\mu_{i}-\mu_{i+1} \neq 2$ for all $1 \leq i \leq r-2$. Suppose that $\mu_{j}-\mu_{j+1}=3$ and $\mu_{j-1}-\mu_{j}=1$ for some $2 \leq j \leq r-1$. Then the node $\left(j-1, \mu_{j-1}-2\right)$ has hook length 4 . If $\mu_{r}$ is 2 , or 3 , then $\mu_{r-1}-\mu_{r} \neq 1$; otherwise the node $(r-1,1)$, respectively $(r-1,2)$, has hook length 4 . This proves one direction of the characterization. The converse is easy.

Using the above characterization of 2-regular and 4-core partitions, and the characterization of simple Specht modules for $p=2$ [17, Main Theorem], we obtain the following corollary.

Corollary 4.6 (of [17, Main Theorem]) Let $p=2$ and $\mu$ be a 2-regular partition. If $\mu$ is a 4 -core then $S^{\mu}$ is simple and $D^{\mu} \cong S^{\mu} \cong Y^{\mu}$.

Corollary 4.7 Let $p=2$ and $\mu$ be a 2-regular partition of $n$. Suppose that $\mu$ is a 4-core. Then any vertex $Q$ of $S^{\mu}$ is conjugate to

$$
\underbrace{V_{1}(2) \times \cdots \times V_{1}(2)}_{w \text { factors }} \hookrightarrow\left(\mathfrak{S}_{2}\right)^{w} \hookrightarrow \mathfrak{S}_{n}
$$

where $w$ is the 2-weight of $\mu$. Furthermore, the complexity of $S^{\mu}$ is $w$.

Proof Let $\mu=2^{k} \mu_{(k)}+\cdots+2 \mu_{(1)}+\mu_{(0)}$ be the 2-adic expansion of $\mu$ with $\left|\mu_{(k)}\right| \neq 0$. We claim that $0 \leq k \leq 1$ and $\mu_{(0)}$ is the 2-core of $\mu$. Note that there are only two types of removable skew 2-hooks, the horizontal 2-hook and the vertical 2hook. Since $\mu_{(0)}$ is obtained from $\mu$ by successively removing all horizontal 2 -hooks it is then not a 2-core if there is some removable vertical 2-hook of $\mu_{(0)}$. In this case, the original partition $\mu$ has two successive non-empty rows whose difference of their sizes is divisible by 2 . However, this is not allowed by the characterization in Lemma 4.5. Thus $\mu_{(0)}=\tilde{\mu}$. It is clear that, in general, if $\mu$ is a $p^{k}$-core for some positive integer $k$ then $\mu_{(l)}=\varnothing$ for all $l \geq k$. 
By Corollary 4.6, the Specht module $S^{\mu}$ is isomorphic to the Young module $Y^{\mu}$. By Theorem 2.6, we deduce that any vertex $Q$ of the Specht module $S^{\mu} \cong Y^{\mu}$ is conjugate to a Sylow 2-subgroup of $\mathfrak{S}_{\rho(\mu)}=\left(\mathfrak{S}_{2}\right)^{\left|\mu_{(1)}\right|}$ where

$$
\left|\mu_{(1)}\right|=\left(n-\left|\mu_{(0)}\right|\right) / 2=(n-|\tilde{\mu}|) / 2=w .
$$

Since the Young module $Y^{\mu}$ has a trivial source; namely the $F Q$-module $F$, by Proposition 2.4 (ii), we have

$$
c_{\mathfrak{S}_{n}}\left(S^{\mu}\right)=c_{Q}(F)=w .
$$

The proof of our corollary is now complete.

The proofs for Theorem 3.2 when $p=2$ and Theorem 3.3 are now clear:

Proof of Theorem 3.2 when $p=2$ We assume all the notation in Theorem 3.2. By Theorem 3.1, the partition $\mu$ is necessarily a 4-core. By Corollary 4.7 , we get $m=$ $w=c$ and $Q$ is conjugate to $\left(V_{1}(2)\right)^{w}$.

Proof of Theorem 3.3 Corollary 4.7 shows that (iii) implies (ii). The implication from (ii) to (i) is trivial. Theorem 3.1 shows that (i) implies (iii). The final statement comes from Corollaries 4.6 and 4.7.

\section{Some applications of the main results}

In [27, Theorem 1], M. Wildon showed that the vertices of an indecomposable Specht module $S^{\mu}$ are non-trivial cyclic if and only if the $p$-weight of $\mu$ is 1 . We have a generalization of this result.

Corollary 5.1 Let $p$ be the characteristic of the field $F$ and suppose that $p \geq 3$. Let $1 \leq m \leq p-1$ be an integer and $\mu$ be a partition. Then a vertex $Q$ of $S^{\mu}$ is abelian of $p$-rank $m$ if and only if the $p$-weight of $\mu$ is $m$. In this case, $Q$ is necessarily elementary abelian.

Proof Assume that $Q$ is abelian. Theorem 3.2 shows that $Q$ is elementary abelian and $m=w$ where $w$ is the $p$-weight of $\mu$. Conversely, suppose that $m=w$. In this case, we have the abelian defect case. Thus $Q$ is necessarily abelian as a subgroup of some defect group of $S^{\mu}$. By Theorem 3.2, $Q$ is has $p$-rank $w=m$.

Remark 5.2 By virtue of [27, Theorem 1], Corollary 5.1 also holds for $p=2$ and $S^{\mu}$ is indecomposable.

There is a special class of Specht modules $S^{\mu}$ whose partition $\mu$ is of the form $\left(\mu_{1}{ }^{a_{1}}, \mu_{2}{ }^{a_{2}}, \ldots, \mu_{r}{ }^{a_{r}}\right)$ where both $\mu_{i}, a_{i}$ are multiples of $p$ for every $1 \leq i \leq r$. We call them the $(p \times p)$-partitions. As another application of our results, we study the vertices of the Specht modules $S^{\mu}$ where $\mu$ is a $(p \times p)$-partition for $p \geq 3$. We 
have seen in Lemma 2.2 that the vertices of an indecomposable module remain unchanged, should the module remains indecomposable upon field extension. Since all Specht modules in the case of $p \geq 3$ are indecomposable, we may assume that $F$ is algebraically closed for the rest of this section.

For $p \geq 3$, Hemmer show that the complexity of the Specht module $S^{\mu}$ when $\mu$ is a $(p \times p)$-partition is strictly less than the $p$-weight of $\mu$ [15, Corollary 1.4]. Thus the following corollary is immediate by applying Theorem 3.2.

Corollary 5.3 Suppose that $p \geq 3$ and $\mu$ is $a(p \times p)$-partition. Then the vertices of $S^{\mu}$ are non-abelian.

Let $E_{p}=V_{1}(p) \times \cdots \times V_{1}(p)$ ( $p$ factors) be the maximal elementary abelian $p$ subgroup of $\mathfrak{S}_{p^{2}}$ as in Theorem 2.7. We have $c_{\mathfrak{S}_{p^{2}}}\left(S^{\left(p^{p}\right)}\right)=c_{E_{p}}\left(S^{\left(p^{p}\right)}\right)=p-1[22$, Theorem 3.1(i)]. Furthermore, for $p \geq 3$, with respect to the generators

$$
(1, \ldots, p),(p+1, \ldots, 2 p), \ldots,\left(p^{2}-p+1, \ldots, p^{2}\right)
$$

of $E_{p}$, a description of the union of irreducible components $W_{p-1}$ of dimension $p-1$ of $V_{E_{p}}^{\#}\left(S^{\left(p^{p}\right)}\right)$ and the vanishing ideal of $W_{p-1}$ is given in [22, Theorem 3.1(ii)]. The vanishing ideal $I$ of $W_{p-1}$ is generated by the element

$$
f\left(x_{1}, \ldots, x_{p}\right)=\left(x_{1} \cdots x_{p}\right)^{p-1} \tilde{f}+\sum_{i=1}^{p} x_{1}^{n(p-1)} \cdots x_{i} \widehat{n(p-1)} \cdots x_{p}^{n(p-1)}
$$

for some homogeneous polynomial $\tilde{f}$ and positive integer $n$, and where the term $x_{1}^{n(p-1)} \cdots x_{i^{n(p-1)}} \cdots x_{p}^{n(p-1)}$ is the product of all $x_{j}^{n(p-1)}$, s with $1 \leq j \leq p$ except the term $x_{i}^{n(p-1)}$. In particular, we have the following lemma.

Lemma 5.4 Any irreducible component of $W_{p-1} \subseteq V_{E_{p}}^{\#}\left(S^{\left(p^{p}\right)}\right)$ is not a hyperplane of $\mathbb{A}^{p}(F)$.

Proof Suppose that $W_{p-1}$ contains a hyperplane, i.e., $f\left(x_{1}, \ldots, x_{p}\right) \in I\left(W_{p-1}\right) \subseteq$ $\left\langle a_{1} x_{1}+\cdots+a_{p} x_{p}\right\rangle$ for some non-zero vector $v=\left(a_{1}, \ldots, a_{p}\right) \in \mathbb{A}^{p}(F)$. Let $g \in$ $F\left[x_{1}, \ldots, x_{p}\right]$ be the polynomial such that

$$
f\left(x_{1}, \ldots, x_{p}\right)=\left(a_{1} x_{1}+\cdots+a_{p} x_{p}\right) g\left(x_{1}, \ldots, x_{p}\right) .
$$

Suppose that at least two of the coordinates of $v$ are non-zero; say $a_{r}$ and $a_{s}$. Let $1 \leq i \leq p$ be such that $i$ is different from either $r$ or $s$. Then there exist elements $b_{1}, \ldots, b_{p}$ of $F$ with $b_{i}=0$, and $b_{j} \neq 0$ for all $1 \leq j \leq p$ and $j \neq i$, such that $a_{1} b_{1}+\cdots+a_{p} b_{p}=0$. Substituting the values $b_{1}, \ldots, b_{p}$ into (5.1), we have the contradiction where

$$
0 \neq b_{1}{ }^{n(p-1)} \cdots \widehat{b_{i}^{n(p-1)}} \cdots b_{p}^{n(p-1)}=0 \cdot g\left(b_{1}, \ldots, b_{p}\right)=0 .
$$

This shows that the coordinates of $v$ are all zero except $a_{j} \neq 0$ for some unique $1 \leq j \leq p$. We deduce that $f\left(x_{1}, \ldots, x_{p}\right)=a_{j} x_{j} g\left(x_{1}, \ldots, x_{p}\right)$ and hence $x_{j}$ is a 
factor of $f\left(x_{1}, \ldots, x_{p}\right)$. However, it is clear from the description of $f$ that $x_{j}$ is not a factor. The proof is now complete.

The next corollary describes the vertices of the Specht module $S^{\left(p^{p}\right)}$ for $p \geq 3$.

Corollary 5.5 For $p \geq 3$, any vertex of $S^{\left(p^{p}\right)}$ is a Sylow $p$-subgroup of $\mathfrak{S}_{p^{2}}$.

Proof Let $Q$ be a vertex of $M=S^{\left(p^{p}\right)}$ and $S$ be a source of $M$. By Mackey Decomposition Theorem, we have

$$
\operatorname{Res}_{E_{p}}^{\mathfrak{S}_{p^{2}}} M \mid \operatorname{Res}_{E_{p}}^{\mathfrak{S}_{p^{2}}} \operatorname{Ind}_{Q}^{\mathfrak{S}_{p^{2}}} S \cong \bigoplus_{g \in E_{p} \backslash \mathfrak{S}_{p^{2}} / Q} \operatorname{Ind}_{g}^{E_{p}}{ }_{Q \cap E_{p}} \operatorname{Res}_{g}^{g} Q{ }_{Q \cap E_{p}}^{g} S
$$

where $E_{p} \backslash \mathfrak{S}_{p^{2}} / Q$ is a set of double coset representatives of $\left(E_{p}, Q\right)$ in $\mathfrak{S}_{p^{2}}$. Suppose that ${ }^{g} Q \cap E_{p} \neq E_{p}$ for all $g$; namely ${ }^{g} Q \cap E_{p}$ is a proper subgroup of $E_{p}$ and hence has order at most $p^{p-1}$. Let

$$
U_{g}=V_{E_{p}}^{\#}\left(\operatorname{Ind}_{g}^{E_{p}}{ }_{Q \cap E_{p}} \operatorname{Res}_{g}^{g} Q \cap E_{p}^{g} S\right) .
$$

In fact, $\operatorname{dim} U_{g}=\operatorname{dim} V_{g}^{\#}{ }_{Q \cap E_{p}}\left(\operatorname{Res}_{g}^{g}{ }_{Q \cap E_{p}}{ }^{g} S\right)$. Since $U_{g}$ is an induced module induced from a proper subgroup of $E_{p}$ we have that the rank variety

$$
U:=V_{E_{p}}^{\#}\left(\operatorname{Res}_{E_{p}}^{\mathfrak{S}_{p^{2}}} \operatorname{Ind}_{Q}^{\mathfrak{S}_{p^{2}}} S\right)=\bigcup U_{g} \subseteq \mathbb{A}^{p}(F)
$$

is a finite union of subvarieties of dimension at most $p-1$. If $U_{g}$ has dimension $p-1$ for some $g$ then it is necessarily that $V_{g}^{\#}{ }_{Q \cap E_{p}}\left(\operatorname{Res}_{g}^{g} Q \cap E_{p}{ }^{g} S\right) \cong \mathbb{A}^{p-1}(F)$ and hence $U_{g}$ is a union of hyperplanes of $\mathbb{A}^{p}(F)$.

Let $N$ be a $k E_{p}$-module such that $N \oplus \operatorname{Res}_{E_{p}}^{\mathfrak{S}_{p^{2}}} M=\operatorname{Res}_{E_{p}}^{\mathfrak{S}_{p^{2}}} \operatorname{Ind}_{Q} \mathfrak{S}_{p^{2}} S$. We have $V_{E_{p}}^{\#}(M) \cup V_{E_{p}}^{\#}(N)=U$. Let $V$ be an irreducible component of $V_{E_{p}}^{\#}(M)$ of dimension $p-1$. By the unique decomposition property of varieties into their irreducible varieties, see [14, Corollary 1.6], we conclude that $V$ is a hyperplane of $\mathbb{A}^{p}(F)$. This contradicts to Lemma 5.4, and hence we conclude that ${ }^{g} Q \cap E_{p}=E_{p}$ for some $g \in \mathfrak{S}_{p^{2}}$; namely $E_{p} \subseteq Q$ up to some conjugation.

Since $S^{\left(p^{p}\right)}$ has complexity $p-1$, different from the $p$-weight of $\left(p^{p}\right)$, by Theorem 3.2 or Corollary 5.3, the vertices cannot be abelian. Thus $Q$ has order strictly larger than $p^{p}$, i.e., $p^{p+1}$ and hence is a Sylow $p$-subgroup of $\mathfrak{S}_{p^{2}}$.

Remark 5.6 The case of $p=2$ is dealt with in [27, Lemma 6]: the Specht module $S^{(2,2)}$ has the Klein four group $V_{2}(2)$ as its vertex, which is different from the description in Corollary 5.5.

\section{Some further questions}

In this section, we include some further questions. The following question is natural following our Theorem 3.3. 
Question 6.1 Given that $\mu$ is $p$-regular and $p \geq 3$, are the vertices of the Specht module $S^{\mu}$ abelian if $\mu$ is a $p^{2}$-core?

To answer Question 6.1, our method used in Sect. 4.2 does not help so much. The main obstruction is that there are Specht modules $S^{\mu}$ which are not Young modules, with $\mu p$-regular and a $p^{2}$-core (for example, the Specht module $S^{(6,3)}$ with $p=3$ ). Readers may have observed that, in order to achieve Theorem 3.3, the main idea is to prove the simplicity of the corresponding Specht modules as in Corollary 4.6. Thus we can partly answer Question 6.1 by imposing this crude assumption.

Proposition 6.2 Let $p \geq 3, \mu$ be a p-regular partition and $Q$ be a vertex of the Specht module $S^{\mu}$. Suppose that $S^{\mu}$ is simple. Let $w$ be a non-negative integer. Then the following statements are equivalent.

(i) The vertex $Q$ is abelian of $p$-rank $w$.

(ii) The vertex $Q$ is elementary abelian of p-rank $w$.

(iii) The partition $\mu$ is a $p^{2}$-core of p-weight $w$.

In any of these cases, the Specht module $S^{\mu}$ has trivial source, complexity $w$ and is a simple Young module.

Proof We only need to show that (iii) implies (i). As we have mentioned in the proof of Corollary 4.7, the $p$-adic expansion of $\mu$ is $\mu_{(0)}+p \mu_{(1)}$, here we allow $\mu_{(1)}=\varnothing$. By the simplicity condition, we deduce that $S^{\mu} \cong Y^{\mu}$ and hence $Q$ is conjugate to a Sylow $p$-subgroup of $\mathfrak{S}_{\rho(\mu)}=\left(\mathfrak{S}_{p}\right)^{\left|\mu_{(1)}\right|}$ by Theorem 2.6. So $Q$ is abelian, necessarily of $p$-rank $w$ and the Specht module $S^{\mu}$ has complexity $w$ by Theorem 3.1 .

Of course, ultimately, we will be delighted to achieve the following goal, if the computation of the vertices of all Specht modules is difficult.

Question 6.3 Classify all indecomposable Specht modules with abelian vertices.

As we have pointed out in the beginning of Sect. 4.2, the technical assumption of 2-regularity is required in Theorem 3.2. The result will be nicer if it works for all indecomposable Specht modules with abelian vertices. The possibility of the existence of an example of a Specht module $S^{\mu}$ with abelian vertices $Q$ which contain $\mathbb{Z}_{4}$ as a direct factor is the main obstruction. We do not have an example of a Specht module that support this possibility of Proposition 4.4.

Question 6.4 For $p=2$, is there an indecomposable Specht module $S^{\mu}$ whose vertices are abelian and contain $\mathbb{Z}_{4}$ as a direct factor?

Acknowledgements The results of this article have been partly achieved during my days in Aberdeen [21, Remark 4.14] and partly done in Singapore. I would like to take the chance to thank Dave Benson and Radha Kessar. I would like to thank Kai Meng Tan for his availability for discussion and careful reading of this article, and Mark Wildon for pointing out Theorem 4.1 and improving an earlier version of Theorem 3.1.

I would like to thank the referees for their valuable suggestions.

Supported by MOE Academic Research Fund R-146-000-135-112. 


\section{References}

1. Adem, A., Milgram, R.J.: Cohomology of Finite Groups, 2nd edn. Grundlehren der Mathematischen Wissenschaften, vol. 309. Springer, Berlin (2004)

2. Alperin, J.L., Evens, L.: Representations, resolutions and Quillen's dimension theorem. J. Pure Appl. Algebra 22, 221-227 (1981)

3. Avrunin, G.S., Scott, L.L.: Quillen stratification for modules. Invent. Math. 66, 277-286 (1982)

4. Benson, D.J.: Representations and Cohomology I, II, 2nd edn. Cambridge Studies in Advanced Mathematics, vol. 30, 31. Cambridge University Press, Cambridge (1990)

5. Bessenrodt, C.: The isomorphism type of an abelian defect group of a block is determined by its modules. J. Lond. Math. Soc. 39(2), 61-66 (1989)

6. Carlson, J.: The varieties and the cohomology ring of a module. J. Algebra 85, 104-143 (1983)

7. Chuang, J., Tan, K.M.: On Young modules of defect 2 blocks of symmetric group algebras. J. Algebra 221, 651-668 (1999)

8. Danz, S., Külshammer, B., Zimmermann, R.: On vertices of simple modules for symmetric groups of small degrees. J. Algebra 320, 680-707 (2008)

9. Donkin, S.: Symmetric and exterior powers, linear source modules and representations of Schur superalgebras. Proc. Lond. Math. Soc. 83, 647-680 (2001)

10. Erdmann, K.: Young modules for symmetric groups. Special issue on group theory. J. Aust. Math. Soc. 71, 201-210 (2001)

11. Feit, W.: The Representation Theory of Finite Groups. North-Holland Mathematical Library, vol. 25. North-Holland, Amsterdam (1982)

12. Grabmeier, J.: Unzerlegbare Moduln mit trivialer Youngquelle und Darstellungstheorie der Schuralgebra. Bayreuth. Math. Schr. 20, 9-152 (1985)

13. Green, J.A.: On the indecomposable representations of a finite group. Math. Z. 70, 430-445 (1959)

14. Hartshorne, R.: Algebraic Geometry. Graduate Text in Mathematics, vol. 52. Springer, New York (1977)

15. Hemmer, D.: The complexity of certain Specht modules for the symmetric group. J. Algebr. Comb. 30, 421-427 (2009)

16. James, G., Kerber, A.: The Representation Theory of The Symmetric Group. Encyclopedia of Mathematics and Its Applications, vol. 16. Cambridge University Press, Cambridge (2009)

17. James, G., Mathas, A.: The irreducible Specht modules in characteristic 2. Bull. Lond. Math. Soc. 31, 457-462 (1999)

18. James, G.: The Representation Theory of the Symmetric Groups. Lecture Notes in Mathematics, vol. 682. Springer, Berlin (1978)

19. James, G.: Trivial source modules for symmetric groups. Arch. Math. 41, 294-300 (1983)

20. Karpilovskii, G.I.: The smallest degree of exact representation of Abelian groups over certain fields of characteristic 0. Sib. Math. J. 11(3), 697-702 (1970)

21. Lim, K.J.: Varieties for Specht modules. PhD Thesis, University of Aberdeen (2009)

22. Lim, K.J.: The varieties for some Specht modules. J. Algebra 321, 2287-2301 (2009)

23. Müller, J., Zimmermann, R.: Green vertices and sources of simple modules of the symmetric group labelled by hook partitions. Arch. Math. (Basel) 89, 97-108 (2007)

24. Murphy, G.M., Peel, M.H.: Vertices of Specht modules. J. Algebra 86, 85-97 (1984)

25. Murphy, G.M.: On decomposability of some Specht modules for symmetric groups. J. Algebra 66, 156-168 (1980)

26. Povzner, A.Ya.: Finding groups of permutations of least degree which are isomorphic to a given Abelian group. Kharkov Mat. Obshch. 14, 151-158 (1937)

27. Wildon, M.: Two theorems on the vertices of Specht modules. Arch. Math. 81, 505-511 (2003)

28. Wildon, M.: Vertices of Specht modules and blocks of the symmetric group. J. Algebra 323, 2243$2256(2010)$ 